\title{
Hidden treasure
}

The auditory system depends on the functional interaction of hair cells (HCs) with their target cells, the spiral ganglia neurons (SGNs). Degeneration of these cells leads to hearing loss, but cell-replacement therapy is a promising treatment strategy. Wei et al. now show that ependymal cells from the walls of the lateral ventricle (LV) can differentiate into cells with biochemical and functional properties similar to those of HCs, and that neural stem cells (NSCs) of the subventricular zone (SVZ) can differentiate into

SGN-like neurons.

First, the authors showed that a subset of cells cultured from the ependymal wall of the mouse LV express the HC marker myosin VIIA. Using bromodeoxyuridine-treated

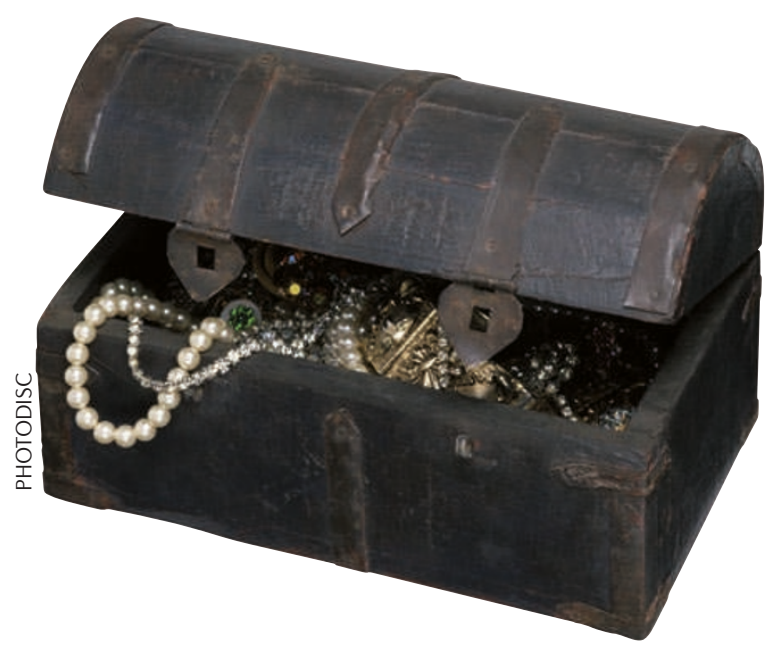

adult mice, the authors confirmed that a number of the polarized ependymal myosin VIIA-positive cells of the LV were proliferating, a prerequisite for selecting cells that might be suitable for replacement therapies. These cells also stained positive for other HC markers, such as ribeye (also known as CTBP2) and myosin VI. Moreover, phalloidin staining revealed actin-based cilia resembling the cilia of hair cells protruding from these cells. Investigating the ependymal layer of the LV in mouse tissue using scanning and transmission electron microscopy revealed that ependymal cells are lined with stereocilia and kinocilia, morphological characteristics of HCs. Moreover, these cells were negative for neuronal or glial protein markers and therefore constitute a distinct epithelial cell type.

To test the functionality and therapeutic potential of these cells, ependymal cells from mice expressing green fluorescent protein under the control of the myosin VIIA promoter were cocultured with SGNs from wild-type mice. Microscopy and electrophysiological investigation revealed that these two cell types had the capacity to form functional synapses. The synaptic marker synapsin I was found to accumulate at the contact sites. These results suggest that ependymal cells and NSCs from the LV have undergone a functional switch.
Next, NSCs from the SVZ were tested for their potential to differentiate into SGNs. As these cells are known to differentiate into glia in a non-neuronal environment, they were pre-differentiated into neurons in culture before being introduced to a cultured organ of Corti in which residual SGNs had been removed. After 1 week in culture, NSCderived neurons had an innervation pattern that strongly resembled that of SGNs in early development. Functional synaptic connections between NSC-derived neurons and HCs were verified by microscopy and electrophysiology. In addition, in cochlear explants that contained SGNs, NSC-derived neurons formed functional connections with deafferated SGNs and seemed to act as interneurons.

Here the authors provide evidence that ependymal and SVZ cells of the adult forebrain might be a viable source of HCs that could be used in replacement therapies for the treatment of hearing loss. One major question is whether the LV-derived HCs can respond to mechanical stimuli such as sounds.

Claudia Wiedemann

ORIGINAL RESEARCH PAPER Wei, D. et al. Cells of adult brain germinal zone have properties akin to hair cells and can be used to replace inner ear sensory cells after damage. Proc. Natl Acad. Sci. USA 105, 21000-21005 (2008) 